Case Report

\title{
NONRECURRENT LARYNGEAL NERVE : A RARE ENTITY
}

\author{
Shrinath D. Kamath P. ${ }^{1}$, Pretty Rathnakar ${ }^{2}$, Kishore Shetty ${ }^{3}$ \\ ${ }^{1}$ Assistant Professor, Department of ENT , K.S. Hegde Medical Academy \\ ${ }^{2}$ Assistant Professor, Department of Anatomy, K.S. Hegde M edical Academy \\ ${ }^{3}$ Professor, Department of ENT, K.S. Hegde Medical Academy \\ Correspondence: \\ Shrinath D. Kamath P. \\ Assistant Professor, Department of ENT, K.S. Hegde Medical Academy, Deralakatte \\ M angalore, Karnataka, India - 575 018. M obile : +91 9980162161 Fax: +91 08242204016 \\ E-mail : drshrinath.kamath@gmail.com
}

\section{Abstract:}

The non recurrent laryngeal nerve (nRLN) is a rare abnormal condition that the head and neck surgeon must aware of in order to avoid postoperative morbidity. High index of suspicion, Preoperative recognition of patients with situs inversus, dysphagia lusoria, identification of medialised vagus nerve and meticulous dissection can minimise the risk of injury to nRLN intraoperatively.

We are reporting a case of nRLN observed during hemithyroidectomy for a 53 year old female patient.

Keywords: non recurrent laryngeal nerve, hemithyroidectomy, vagus nerve, situs inversus, dysphagia lusoria.

\section{Introduction :}

The nonrecurrent laryngeal nerve (nRLN) is a rare anatomical anomaly. The incidence is variously reported to be between 0.3 and $1 \%[1]$ on the right side and extremely rare on the left side $(0.004 \%)$ [2] The right $n R L N$ is associated with a right subclavian artery arising directly from the aortic arch. The left $n R L N$ is associated with situs inversus [3,4] or loss of ductus arteriosus during the foetal life [1].We report this case to emphasise the fact that $n R L N$ is a rare occurrence and it should always be taken in to consideration during thyroidectomy in order to avoid accidental injury.

\section{Case Report:}

A thirty eight years old female presented to us with a painless anterior neck mass since last 4 years which is gradually progressing in size. On clinical examination, the right lobe of the thyroid was found to be enlarged with a solitary nodule measuring about 5 by $4 \mathrm{cms}$ and was firm in consistency. Ultrasound revealed a solitary nodule in the right lobe and the FNAC showed colloid goitre. An indirect laryngoscopic examination showed normal vocal cord function. After confirming that the patient is euthyroid she was subjected to right hemithyroidectomy.

During the surgery we could not identify the right recurrent laryngeal nerve (RLN) at the tracheooesophageal groove. Normally it forms one of the boundaries of Beahr's triangle, the other two sides being formed by common carotid and inferior thyroid artery [5].However careful exploration caudally revealed a nerve, running medially in to the cricothyroid membrane parallel to the inferior thyroid artery. When the nerve was traced proximally it was found to be originating from the cervical vagus, thus confirming itsidentity.

The patient was subjected to barium swallow postoperatively to look for the anomalous right subclavian artery. The barium swallow didn't show an indentation on the oesophagus.

\section{Discussion :}

The relationship of the thyroid gland to the recurrent laryngeal nerve is of major surgical significance because damage to the nerve leads to disability in phonation or difficulty in breathing. nRLN, a rare anomaly, passes transversely into larynx with or without its recurrent branches directly arising from the vagus nerve which is very vulnerable during thyroid surgery[6].

Steadman was the first to observe a nRLN in a cadaver in 1863 who also noted then an anomaly of the origin and course of the right subclavian artery. He concluded that the 
vascular anomaly described earlier by Hunauld in 1785, was closely associated with the nRLN. [7]

In a study by Li $X$ et al 821 recurrent laryngeal nerves were anatomized during 2496 thyroid operations, from which 5 were confirmed to hold non-recurrent laryngeal nerves $(0.61 \%) .[6]$

Henry JF et al reported 33 cases of nonrecurrent inferior laryngeal nerves in 6307 cervicotomies for thyroid and parathyroid excision (0.52\%). The anomaly was observed in 31 cases from 4921 dissections on the right side (0.63\%) and in two cases from 4673 dissections on the left side $(0.04 \%) .[3]$

The nRLN was encountered in a study only after performing 585 thyroid operations in three years with an overall incidence of $0.2 \%[8]$. Others have reported a small recurrent laryngeal nerve with a major non-recurrent trunk. $[9,10]$

The origin of the $\mathrm{nRLN}$ is cervical. Three types can be distinguished: type $1 \mathrm{nRLN}$ arises directly from the vagus and runs together with the vessels of the superior thyroid pedicle; type $2 \mathrm{~A}$ nRLN follows a transverse path parallel to and over the trunk of the inferior thyroid artery; and type $2 \mathrm{~B} \mathrm{nRLN}$ follows a transverse path parallel to and under the trunk or between the branches of the inferior thyroid artery[4].We report type $2 \mathrm{AnRLN}$.

The preoperative diagnosis of a nRLN is extremely difficult.A left nRLN has been suspected in patients with proved situs inversus. The presence of situs inverssus on a preoperative chest $x$ ray should alert the surgeon to the possibility of a left sided nRLN. A right sided nRLN should be suspected preoperatively in those patients with dysphagia lusoria due to an aberrant retro oesophageal right subclavian artery.[3,11].The retroesophageal subclavian artery may be detected on chest $\mathrm{x}$-ray films (20\%) or by the compression and distortion of the esophagus shown during barium swallow tests (97\%)[3].

Intraoperatively, the best way to avoid morbidity is to identify the nerve with a systematic dissection based on usual anatomical landmarks.Presence of a nRLN should be suspected when the nerve is not found at the normal site. In such cases any transversely running structure between the carotid sheath and the larynx should not be cut without meticulous dissection. Further vagus trunk sited medially to the common carotid artery, has been reported. This is due to the fact that the nRLN is shorter than the RLN and the traction on the laryngo tracheal axis during thyroidectomy leads to the medialisation of vagus[12].

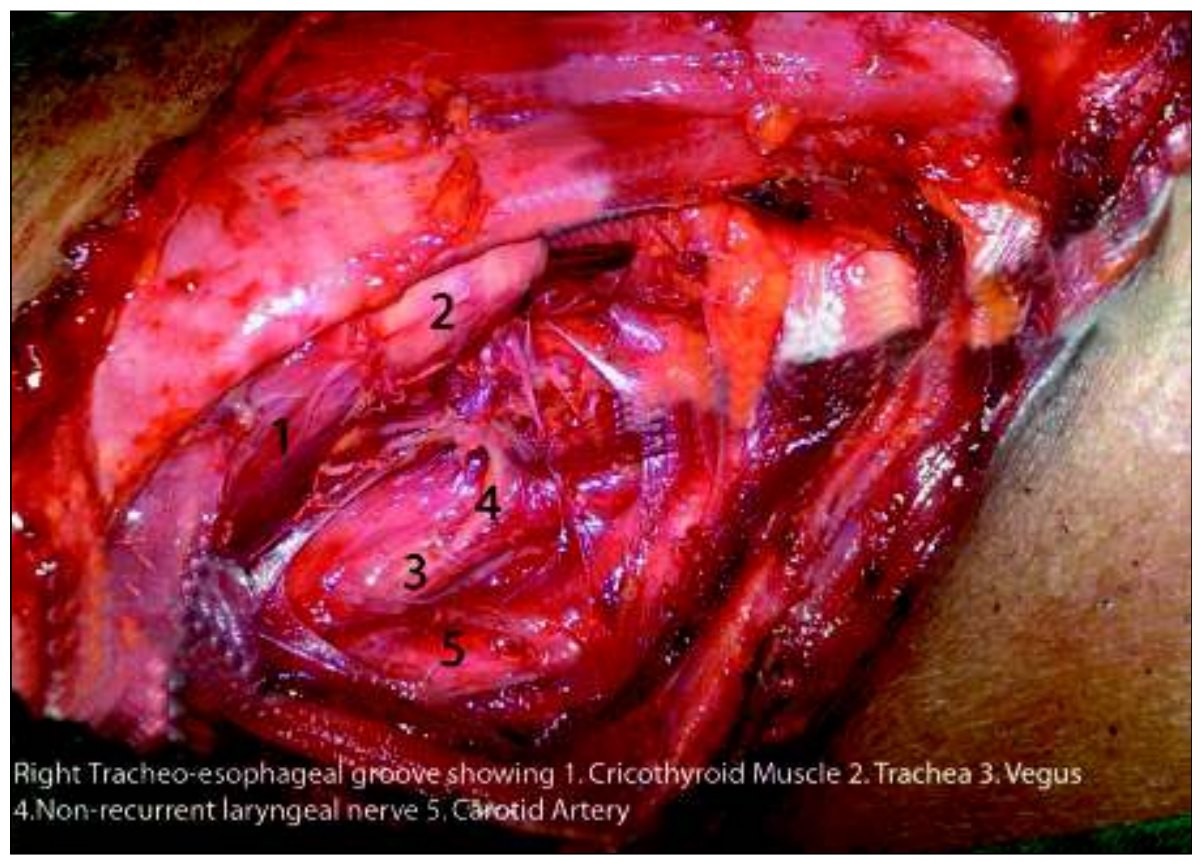




\section{Conclusion :}

1. The incidence of $n R L N$ is very rare but when present, it is most vulnerable to injury during thyroidectomy.

2. Preoperative diagnosis of situs inversus and dysphagia lusoria may hint towards its existence.

\section{References:}

1. Neil Weir.1996.Anatomy of larynx and tracheobroncheal tree; Scott Brown textbook of Otorhinolaryngology, $6^{\text {th }} \mathrm{Ed}$, vol 1, Oxford: Buttrworth Heinemann. 1/12/1- 1/2/28.

2. Mehmet Uludag Adnan Isgor, Anatomic variations of the nonrecurrent inferior laryngeal nerve, , BMJ Case Reports 2009; doi:10.1136/bcr.10.2008.1107

3. Henry JF, Audiffret J,Denizot $A$, et al.The nonrecurrent inferior laryngeal nerve: review of 33 cases, including two on the left side. Surgery 1988; : 977-84.

4. Toniato A, Mazzarotto $R$, Piotto $A$, et al. Identification of the nonrecurrent laryngeal nerve during thyroid surgery: 20-year experience. World J Surg 2004; :659-61.

5. John C Watkin,Mark N Gaze,Janet A Wilson.2000.Tumours of the thyroid and parathyroid glands :Stell and Maran's Head and Neck Surgery. $4^{\text {th }}$ Ed, Oxford: Butterworth, Heinemann, 459 - 486.

6. Li X, Wang Z, Lu X, Li J, Huang Y, Huang J, Long X. Non-recurrent laryngeal nerve related to thyroid surgery: a report of 5 cases and literature review. 2010 Jun;16(6):CS71-5. PM ID:20512096 [PubM ed indexed for M EDLINE]
3. Awareness of its existence and correct surgical technique will minimise the morbidity.

4. Medialisd vagal trunk may give a clue about $n R L N$ intraoperatively.

7. Steadman. GW. A singular distribution of some of the Delves and arteries of the neck and the top of the thorax. M in M ed Surg] 1823; 19, 564-65.

8. Non-recurrent Laryngeal Nerve in Thyroid Surgery A N Hisham (MS), A Sarojah (MS), A Alvin (MS), nt'east and Endoet'ine Surgical Unit, Department of Surget-y, Kuala Lumpur Hospital, Kuala Lumpur, M alaysia. MedJ Malays;a Val 56 No 4 Dec 2001.500-2

9. Sanders G, Uyeda R, Karlan M. Nonrecurrent inferior lalyngeal nerves and their association with a recurrent branch. The American J Surg 1983; 146, 501-3.

10. Proye CA, Carnaille EM, Goropoulos A. Nonrecurrent and recurrent inferior laryngeal nerve. A surgical pitfall in cervical exploration. The Am] Surg 1991; 162, 495-96.

11. Coady M, Adler F, Davila J, et al. Non recurrent laryngeal nerve during carotid artery surgery: case report and literature review. J Vasc Surg 2000;32:192-6.

12. A Toniato,I Merante Boschin,C Pagetta,E Casalide and M Pelizzo.A "Pilot light" of the right recurrent laryngeal nerve ,Acta Otorhinolaryngol Ital. 2010 April; 30(2): 107-109. 